\title{
To the rescue-migrating renin lineage cells heal the injured glomerular mesangium
}

In a new study published in the Journal of the American Society of Nephrology, Charlotte Starke, Christian Hugo and colleagues from the Technische Universität Dresden, Germany, report that extraglomerular renin-expressing cells can migrate into the glomerulus and reconstitute the injured mesangium. Mesangiolysis is a component of many chronic kidney diseases and is a major cause of glomerular dysfunction.

\section{4 ...cells of renin lineage} \section{replenish the mesangial} compartment in the glomerular tuft... 97

On the basis of previous data from a rat model of mesangial proliferative nephritis (the anti-Thyl model), the researchers hypothesized that cells from the juxtaglomerular apparatus might regenerate the intraglomerular mesangium. However, the exact origin and type of such cells was unclear. "Since renin cells are known to be precursors for multiple cell types during nephrogenesis, including the mesangial cells, we thought these cells might represent a source for repopulating cells after mesangial injury," explains Starke.

The team generated an inducible transgenic mouse line in which tetracycline administration enables LacZ-labelling of renin-lineage cells. Mesangial injury was selectively induced with antimesangial cell serum and lipopolysaccharide. After 10 days, the researchers harvested kidney biopsy samples for study.

They found that at least $25 \%$ of glomerular tufts in injured mice, but none in the healthy controls, contained LacZexpressing cells. Interestingly, these cells no longer expressed renin, suggesting that the renin-expressing progenitor cells undergo differentiation-recapitulating mechanisms of glomerulogenesis. In addition, these LacZ-positive cells expressed the known mesangial markers a8-integrin and platelet-derived growth factor receptor $\beta$, and not markers of podocytes, endothelial or parietal epithelial cells.

"Our data demonstrate for the first time that cells of renin lineage replenish the mesangial compartment in the glomerular tuft after selective mesangial injury," Starke points out. Currently, the team is planning further studies to investigate the mechanisms behind this process. "For instance, we are interested in the signals produced by the injured mesangial cells to attract potential precursors," concludes Starke.

Clemens Thoma

Original Article Starke, C. et al. Renin lineage cells repopulate the glomerular mesangium after injury. J. Am. Soc. Nephrol. doi:10.1681/ASN.2014030265 